\title{
Long Bone Phenotypic Analyzes Of A Rank Transgenic Mouse Line
}

Baaroun V. ${ }^{1}$, . Coudert A. ${ }^{1}$, Marty C. ${ }^{2}$, Castaneda B. ${ }^{1}$, Berdal A. ${ }^{1}$, Descroix V. ${ }^{1}$

${ }^{1}$ Laboratoire de Physiopathologie Orale Moléculaire - Equipe 5 - Centre de Recherche des Cordeliers - UMRS872 - Paris;

${ }^{2}$ Laboratoire Os et Articulations - Hôpital Lariboisière - UMRS 606 - Paris

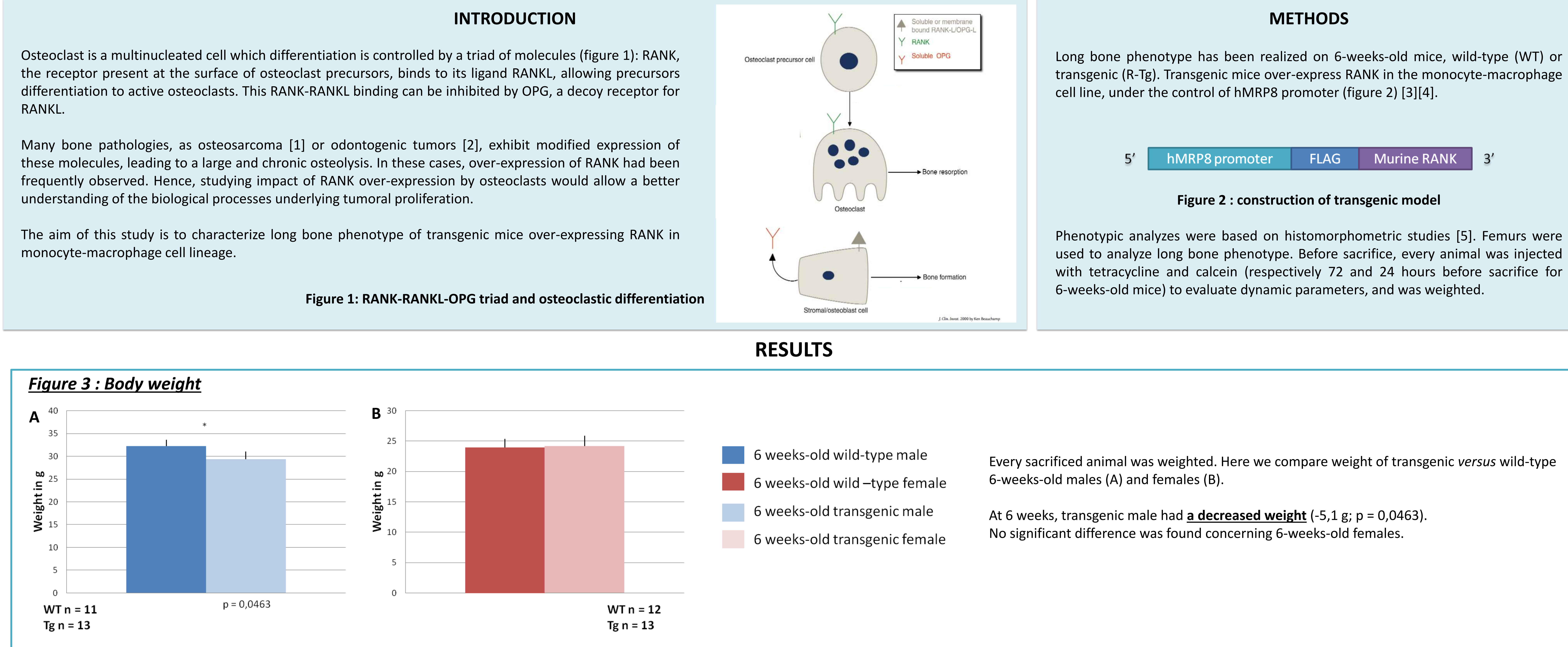

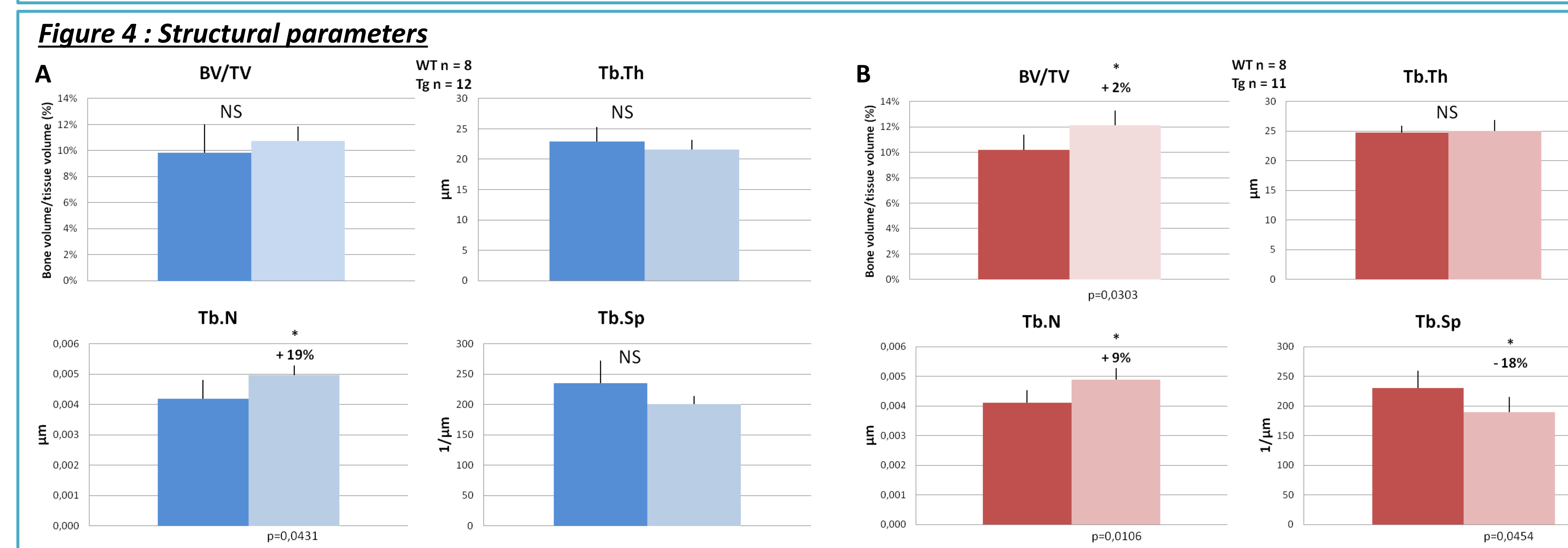

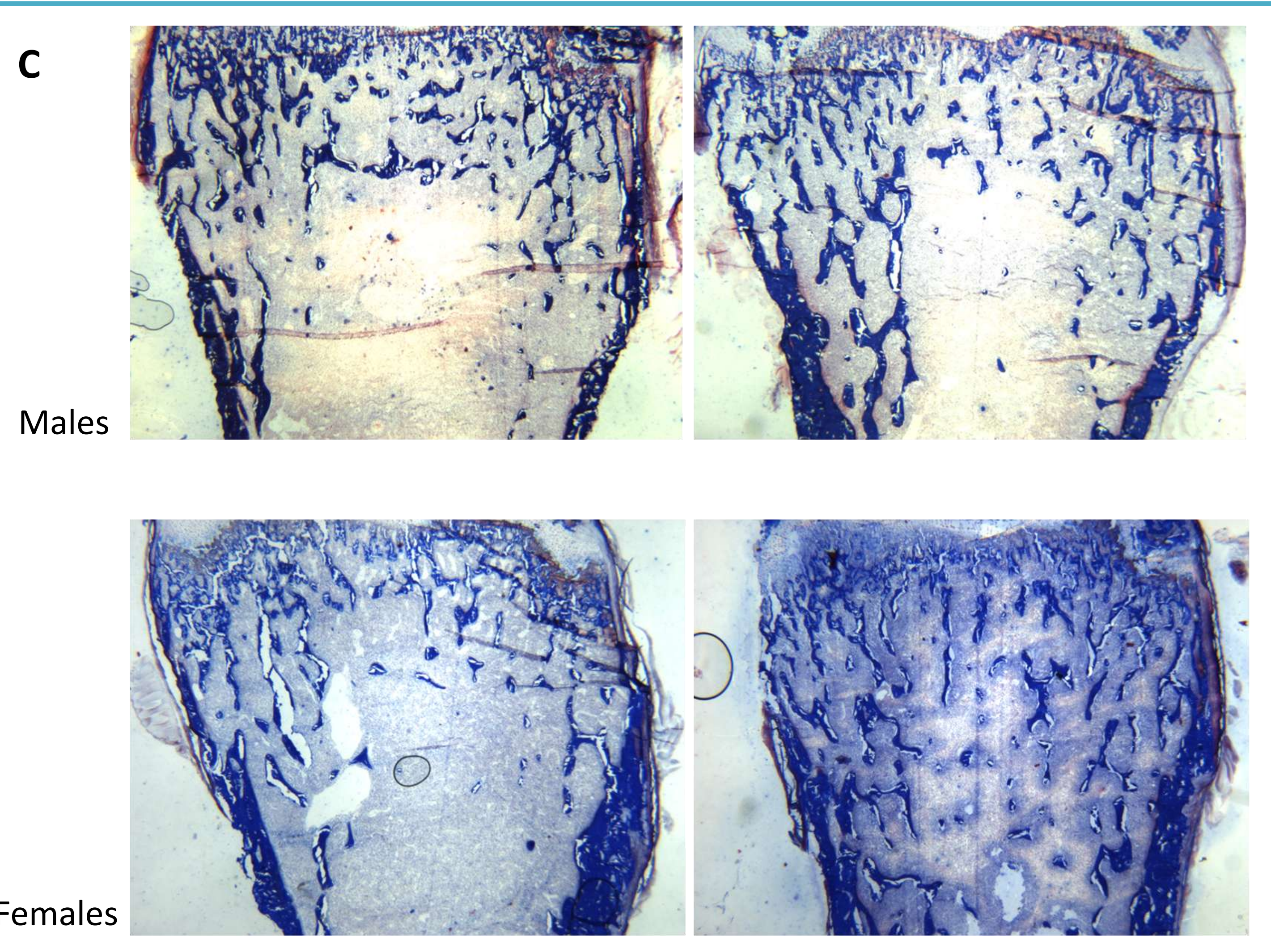

Measures of bone volume (BV/TV), trabecular thickness (Tb.Th), trabecular number (Tb.N) and trabecular separation (Tb.Sp) of 6-weeks-old males (A) and females (B). C: blue aniline staining of 6-weeks-old WT (left) and R-Tg (right) males and females (objective $\times 5$ )

Transgenic males had an increased trabecular number $(+19 \% ; p=0,0431)$.

Transgenic females had an increased bone volume $(+2 \% ; p=0,0303)$, an increased trabecular number $(+19 \% ; p=0,0106)$ and a decreased trabecular separation $(-18 \% ; p=0,0454)$

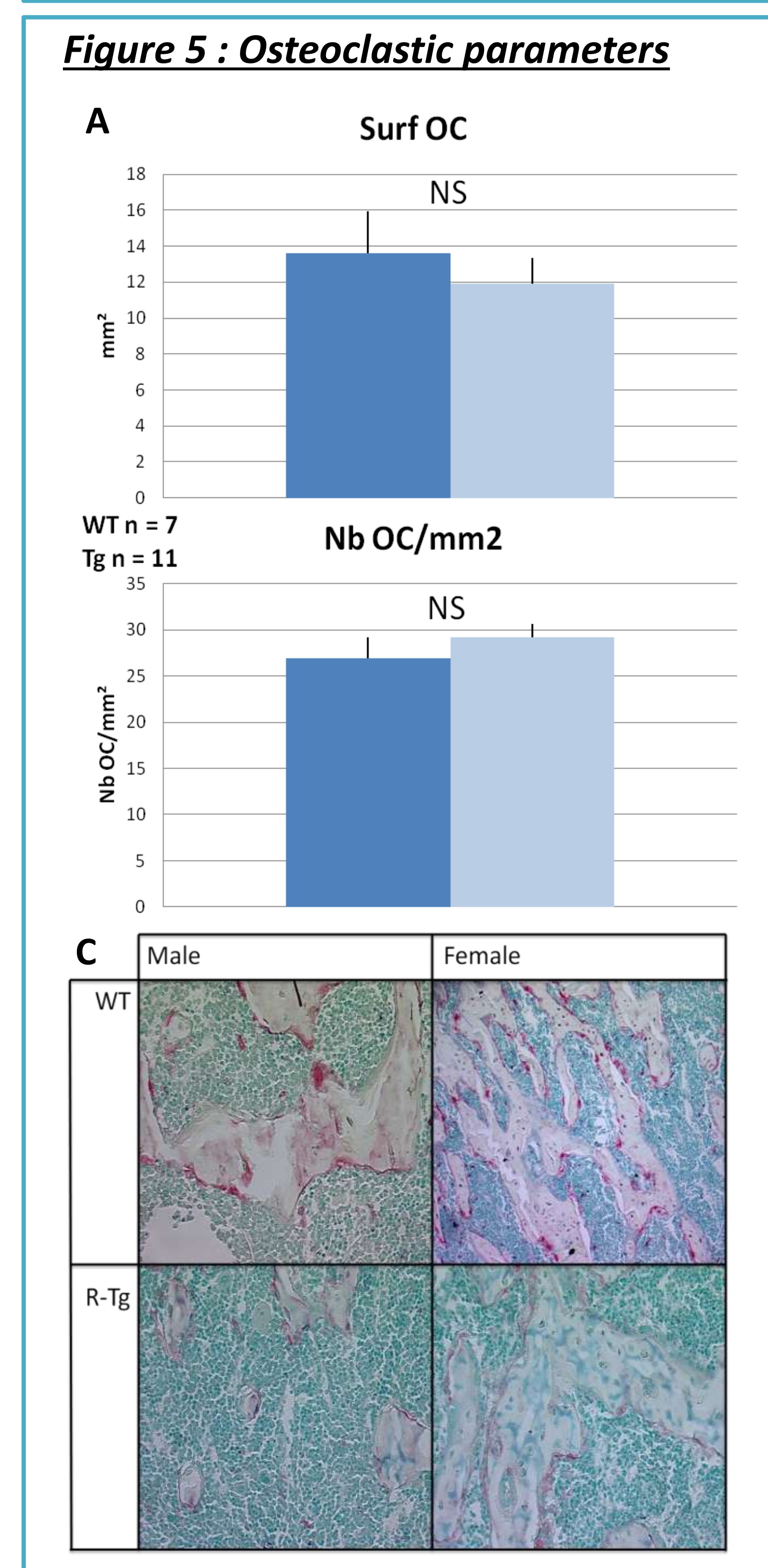

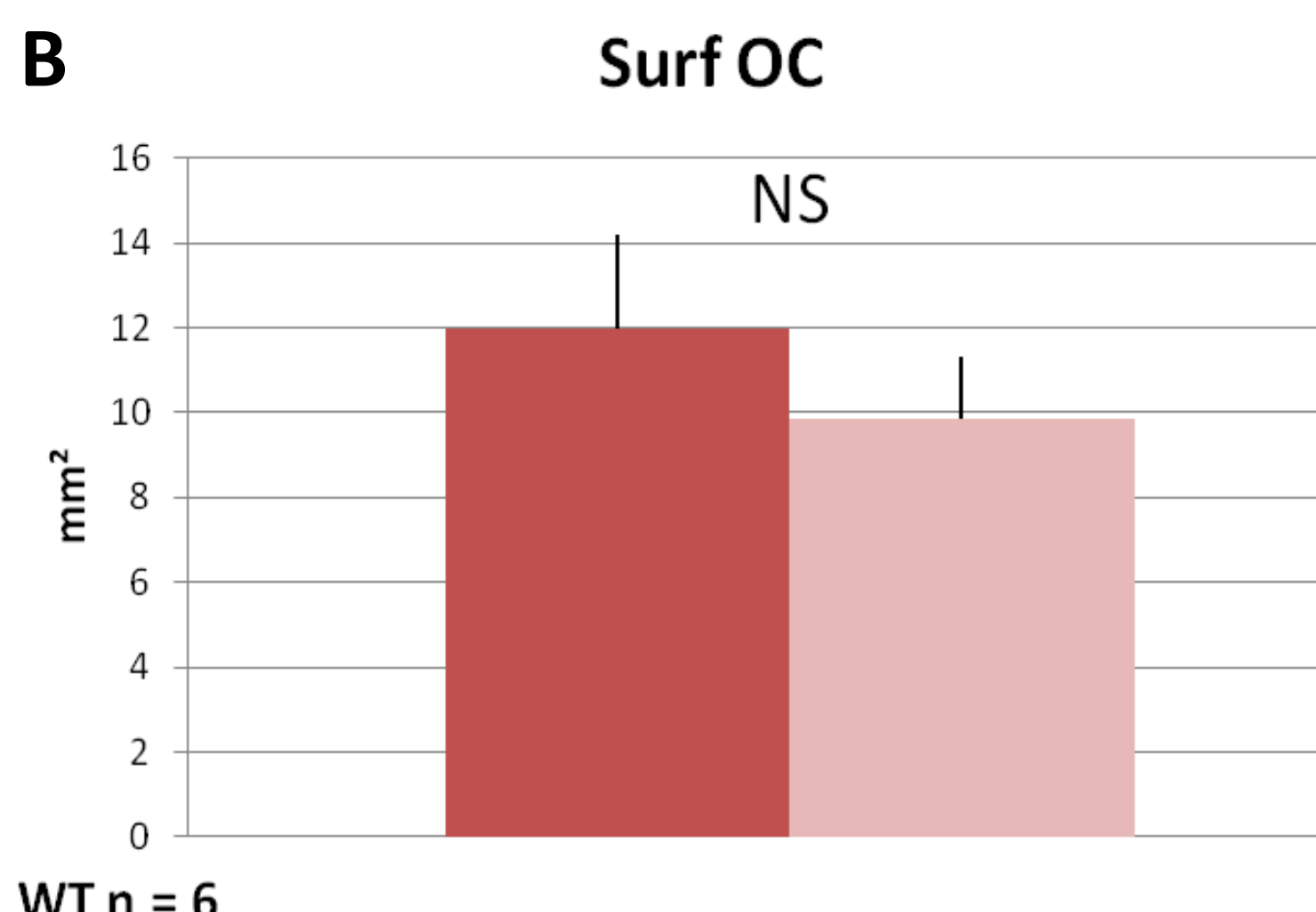

$\mathrm{WT} n=6$
$\operatorname{Tg} n=12$

$\mathrm{Nb}$ OC/mm2

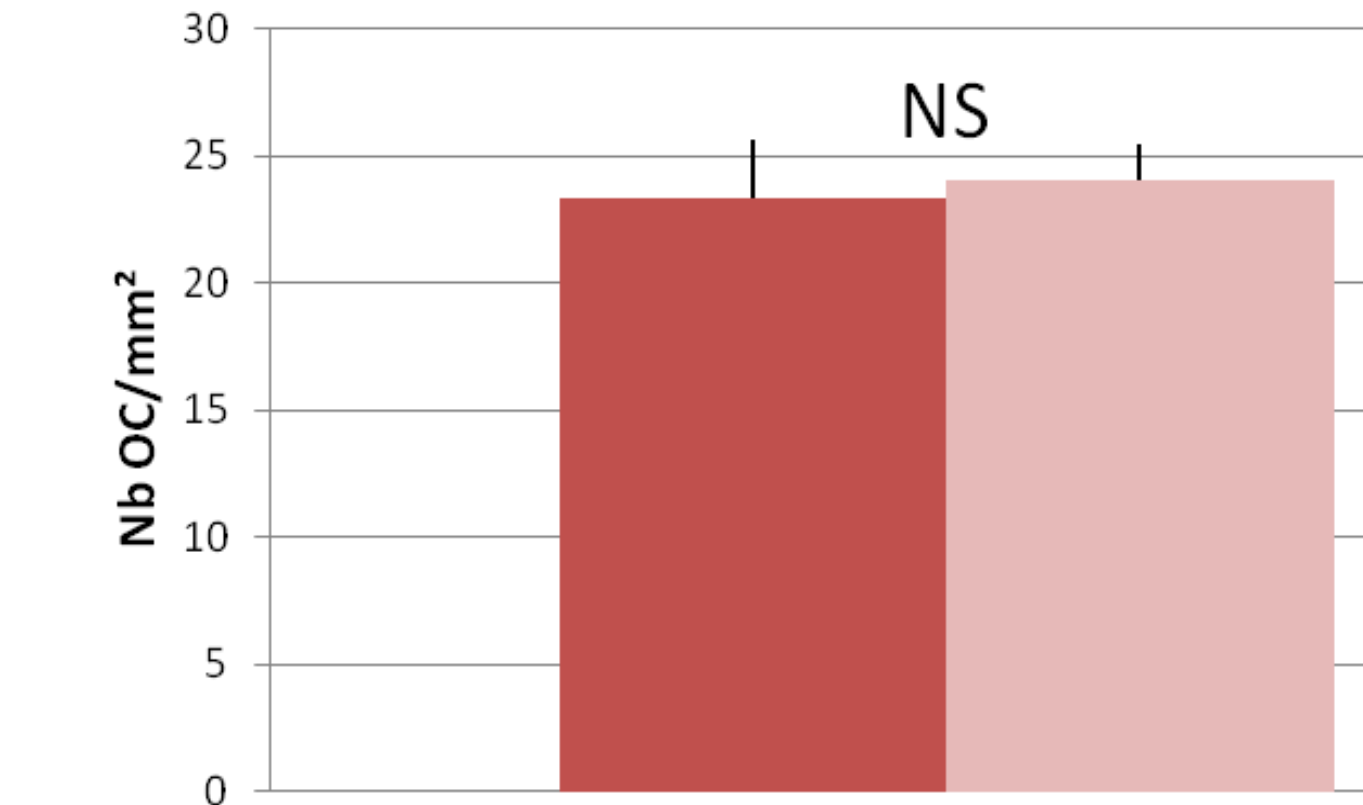

$A$ and B: Comparison of osteoclastic surface (Surf OC) and number of osteoclasts $\left(\mathrm{Nb} \mathrm{OC} / \mathrm{mm}^{2}\right)$ at 6 weeks.

C: TRAP staining (contra-stained with green methyl; objective x 25).

No significant difference was found between groups.

\section{Figure 6: Dynamic parameters}

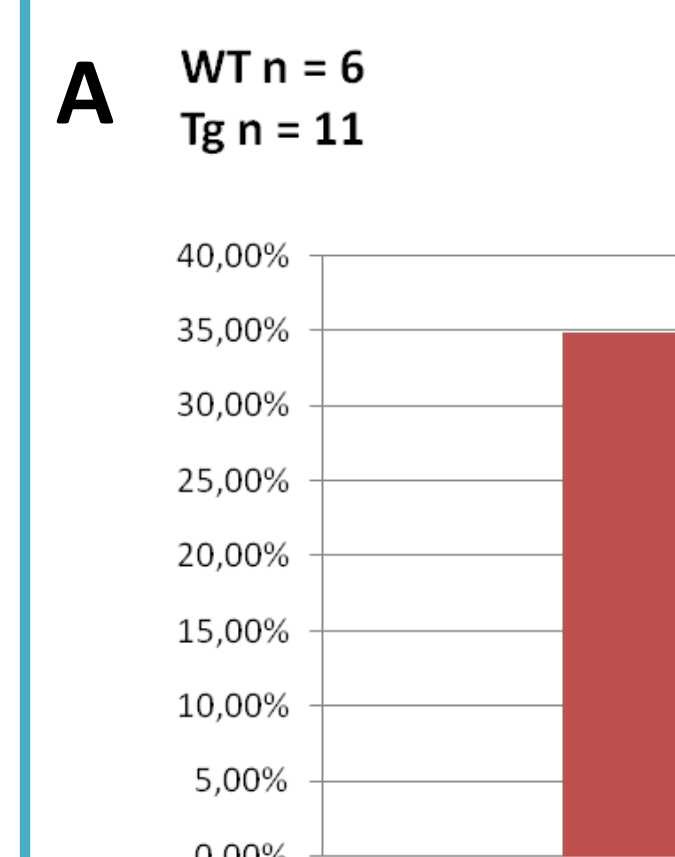

Ms

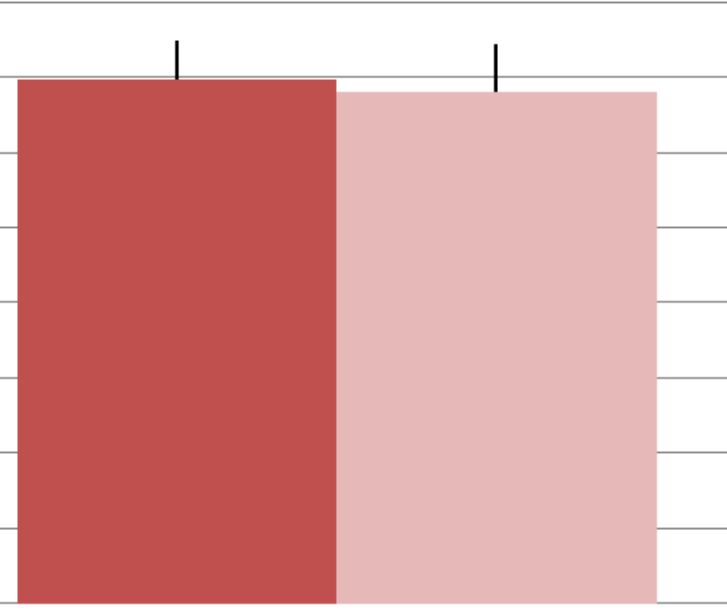

MAR

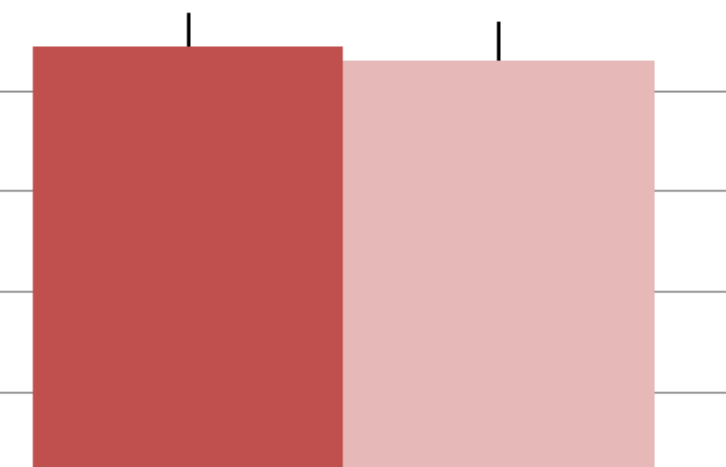

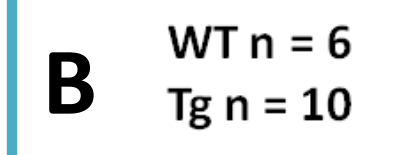

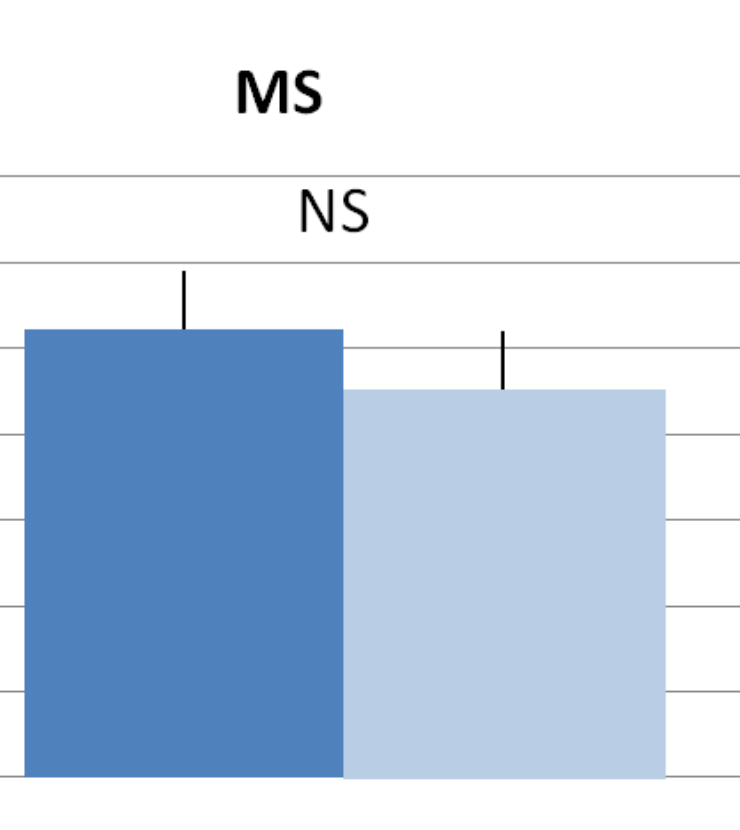

MAR
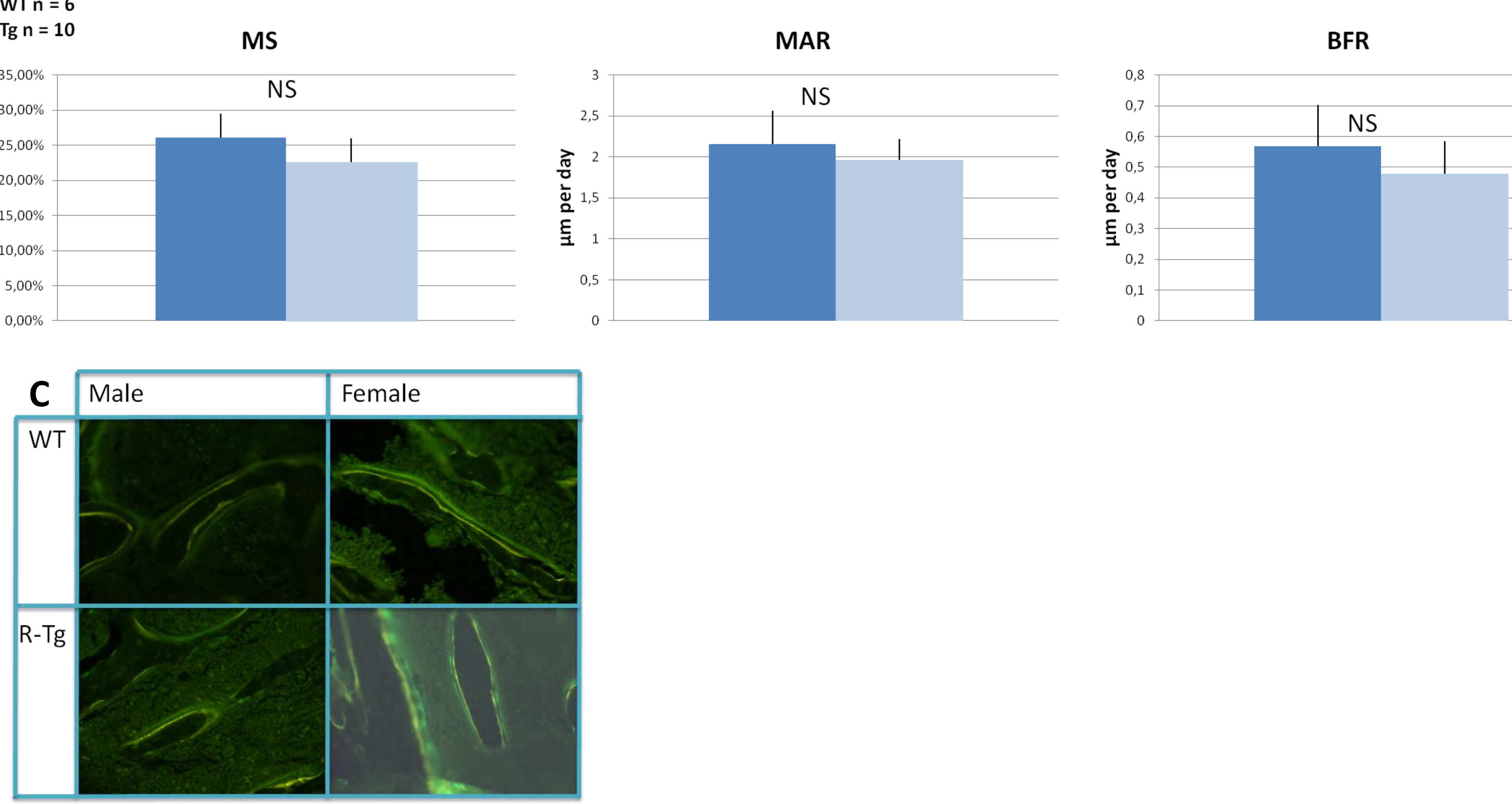

Measure of mineralizing surfaces (MS), mineral apposition rate (MAR) and bone formation rate (BFR) for 6-weeks-old males (A) and females (B).

$C$ : fluorescence microscopy observation of tetracycline and calcein signal (objective x 25 ).

No significant difference was found between different groups.

\section{CONCLUSION}

Those preliminary results of phenotypic analyses show some differences between male and female, WT and R-Tg. Transgenic males exhibit a decreased body weight and an increased Tb.N, whereas transgenic females have an increased BV/TV, Tb.N and a decreased Tb.Sp. No significant difference was found concerning osteoclastic and dynamic parameters.

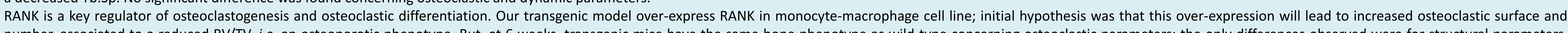
Fife ic males seem to exhibit almost no long bone phenotype, whereas females have a slightly more marked phenotype. Those differenc

of jaw bone, in order to find if this RANK over-expression has different effects on different types of bone. We will also study femurs and jaws at different ages, specially 2-weeks-old, since this age

[1] Mori K et al. Osteosarcoma: current status of immunotherapy and future trends. Oncol Rep. 2006 Mar;15(3):693-700.

[2] Ruhin B et al. Facts and Hypothesis on Osteolytic Lesions Related to Normal and Tumoral Epithelial Dental Cell Differentiation. In. Bone Cancer: Progression and Therapeutic Approaches. Auteur: Heyman D. Editions Elsevier. 2009. [3] Castaneda B et al. Bone resorption control of tooth eruption and root morphogenesis: Involvement of the receptor activator of NF-KB (RANK). J Cell Physiol. 2011 Jan;226(1):74-85.

[4] Duheron V et al. Receptor activator of NF-kappaB (RANK) stimulates the proliferation of epithelial cells of the epidermo-pilosebaceous unit. Proc Natl Acad Sci U S A. 2011 Mar 29;108(13):5342-7.

[5] Parfitt AM et al. Bone histomorphometry : standardization of nomenclature, symbols, and units. Report of the ASBMR Histomorphometry Nomenclature Committee. J Bone Miner Res. 1987; 2(6):595-610,

[6] Robinson L et al. Estrogen inhibits RANKL-stimulated osteoclastic differentiation of human monocytes through estrogen and RANKL-regulated interaction of estrogen receptor-alpha with BCAR1 and Traf6. Exp Cell Res. 2009 Apr 15;315(7):1287-301. 